ARTICLE

\title{
External stimulation-controllable heat-storage ceramics
}

Hiroko Tokoro ${ }^{1,2,3}$, Marie Yoshikiyo', Kenta Imoto', Asuka Namai', Tomomichi Nasu1, Kosuke Nakagawa', Noriaki Ozaki ${ }^{1}$, Fumiyoshi Hakoe, Kenji Tanaka', Kouji Chiba ${ }^{4}$, Rie Makiura ${ }^{5,6}$, Kosmas Prassides ${ }^{7}$ \& Shin-ichi Ohkoshi ${ }^{1,2}$

Commonly available heat-storage materials cannot usually store the energy for a prolonged period. If a solid material could conserve the accumulated thermal energy, then its heatstorage application potential is considerably widened. Here we report a phase transition material that can conserve the latent heat energy in a wide temperature range, $T<530 \mathrm{~K}$ and release the heat energy on the application of pressure. This material is stripe-type lambda-trititanium pentoxide, $\lambda-\mathrm{Ti}_{3} \mathrm{O}_{5}$, which exhibits a solid-solid phase transition to beta-trititanium pentoxide, $\beta-\mathrm{Ti}_{3} \mathrm{O}_{5}$. The pressure for conversion is extremely small, only 600 bar $(60 \mathrm{MPa})$ at ambient temperature, and the accumulated heat energy is surprisingly large $\left(230 \mathrm{~kJ} \mathrm{~L}^{-1}\right)$. Conversely, the pressure-produced beta-trititanium pentoxide transforms to lambda-trititanium pentoxide by heat, light or electric current. That is, the present system exhibits pressure-and-heat, pressure-and-light and pressure-and-current reversible phase transitions. The material may be useful for heat storage, as well as in sensor and switching memory device applications.

\footnotetext{
${ }^{1}$ Department of Chemistry, School of Science, The University of Tokyo, 7-3-1 Hongo, Bunkyo-ku, Tokyo 113-0033, Japan. ${ }^{2}$ CREST, JST, K's Gobancho, 7 Gobancho, Chiyoda-ku, Tokyo 102-0076, Japan. ${ }^{3}$ Division of Materials Science, Faculty of Pure and Applied Sciences, University of Tsukuba, 1-1-1 Tennodai, Tsukuba, 305-8577, Japan. ${ }^{4}$ Science and Technology System Div., Ryoka Systems Inc., Tokyo Skytree East Tower, 1-1-2 Oshiage, Sumida-ku, Tokyo 131-0045, Japan. ${ }^{5}$ Nanoscience and Nanotechnology Research Center, Osaka Prefecture University, 1-2 Gakuen-cho, Naka-ku, Osaka 599-8570, Japan. ${ }^{6}$ PRESTO, JST, 4-8-1 Honcho, Kawaguchi 332-0012, Japan. 7 WPI-Advanced Institute for Materials Research, Tohoku University, 2-1-1 Katahira, Sendai 980-8577, Japan. Correspondence and requests for materials should be addressed to S.O. (email: ohkoshi@chem.s.u-tokyo.ac.jp).
} 
$\mathrm{P}$ hase transition phenomena, such as metal-insulator, ferroelectric ferromagnetic, and spin transitions, are attractive issues in the fields of physics, chemistry and materials science. Phase transitions are controlled not only by temperature change but also by other external stimuli such as pressure, light-irradiation or electric current flow. For example, for pressure-induced phase transitions, pressure-induced metal-semiconductor transition in a molybdenum disulphide ${ }^{1}$, pressure-induced superconductor transition in a fulleride ${ }^{2}$ and pressure-induced ferroelectric-antiferroelectric transition in a perovskite system ${ }^{3}$ have been reported. For light-induced phase transitions, light-induced crystalline-amorphous transitions in chalcogenides $^{4,5}$, light-induced metal-semiconductor transition in a trititanium pentoxide ${ }^{6}$ and insulator-metal transition in perovskite manganites ${ }^{7,8}$, light-induced spin-crossover transitions in metal complexes ${ }^{9-12}$ and light-induced charge-transfer transition in organic molecules ${ }^{13,14}$ and metal complexes ${ }^{15}$ have been reported. Furthermore, for current-induced phase transitions ${ }^{16-18}$, current-induced insulator-metal transition in organic compound and current-induced magnetic-domain-wall switching in gallium manganese arsenide have been reported.

In recent years, heat-storage materials have been attracting attention from the viewpoint of energy saving. Development of high-performance heat-storage materials is important for the effective use of waste heat from blast furnaces in factories. Phase transition materials are considered to be useful as latent heat-storage materials. These are divided into solid-liquid and solid-solid phase transition types. In the former, the phase transition at the melting point (m.p.) is used for the heat storage. For example, water $\left(320 \mathrm{~kJ} \mathrm{~L}^{-1}\right.$ at m.p. $\left.=0{ }^{\circ} \mathrm{C}\right)$, paraffin $\left(140 \mathrm{~kJ} \mathrm{~L}^{-1} \text { at m.p. }=64^{\circ} \mathrm{C}\right)^{19}$ and polyethylene glycol $\left(165 \mathrm{~kJ} \mathrm{~L}^{-1} \text { at m.p. }=20^{\circ} \mathrm{C}\right)^{20}$ are known. In these cases, there are concerns of liquid spill from the system and mixing (or reaction) with the surrounding media. From this angle, a solidsolid phase transition material is stiff and its form is maintained without support, while at the same time it has chemical stability against the surrounding media. Well-known solid-solid phase transition materials for heat-storage usage include copolymers (for example, hyperbranched polyurethane: $150 \mathrm{~kJ} \mathrm{~L}^{-1}$ at $\left.67^{\circ} \mathrm{C}\right)^{21}$, organic compounds (for example, neopentylglycol: $165 \mathrm{~kJ} \mathrm{~L}^{-1}$ at $48^{\circ} \mathrm{C}$ and pentaerythritol: $360 \mathrm{~kJ} \mathrm{~L}^{-1}$ at $\left.188^{\circ} \mathrm{C}\right)^{22,23}$ and organometallic compounds (for example, bis $\left(n\right.$-hexadecylammonium) tetrachlorozincate: $120 \mathrm{~kJ} \mathrm{~L}^{-1}$ at $103^{\circ} \mathrm{C}$ and bis(n-decylammonium) tetrachlorocuprate: $60 \mathrm{~kJ} \mathrm{~L}^{-1}$ at $\left.34^{\circ} \mathrm{C}\right)^{19,24,25}$. In general, such phase change heatstorage materials cannot store the energy for a prolonged period below the phase transition temperature. If a solid material could conserve the accumulated thermal energy and release it only on demand, then its heat-storage application potential is considerably widened. From this angle, our work focused on a phase transition where the latent heat of thermal phase transition could be stored.

In this paper, we report a heat-storage material composed of lambda-trititanium pentoxide. The solid-solid phase transition of this material can be controlled by heat, pressure application, light-irradiation and current flow. This heat-storage material can conserve a high accumulation of energy and release it by the application of a remarkably small external pressure.

\section{Results}

Material and morphology. The sample of the titanium oxide, a new series of lambda-trititanium pentoxide $\left(\lambda-\mathrm{Ti}_{3} \mathrm{O}_{5}\right)$, was produced by sintering rutile- $\mathrm{TiO}_{2}$ particles in a hydrogen atmosphere (see Methods). Elemental analysis using inductively coupled plasma mass spectrometry confirms that the formula of the material is $\mathrm{Ti}_{3} \mathrm{O}_{5}$. Scanning electron microscopy (SEM) and transmission electron microscopy (TEM) images of the obtained sample show a coral-like morphology with particle size of $\sim 4 \times 1 \mu \mathrm{m}$ (Supplementary Fig. 1), composed of aggregates of rectangular-shaped nanorods, of which the majority are $\sim 200 \times 30 \mathrm{~nm}$ dimensions (hereafter called 'stripe-type- $\lambda$ $\mathrm{Ti}_{3} \mathrm{O}_{5}$ ', Fig. 1a). The high-resolution TEM (HRTEM) image is shown in Fig. 1b. The Fourier transform analysis of the HRTEM image showed that the growth direction of the nanorods is along the crystallographic $b$ axis. The atomic level image from HRTEM corresponds to the visualized electron density distribution map on the $b c$ plane calculated by the maximum entropy method (MEM; Fig. 1c), described later.

Pressure-induced phase transition. X-ray powder diffraction (XRPD) measurements were performed to investigate the pressure $(P)$ dependence of the crystal structure of the stripetype- $\lambda-\mathrm{Ti}_{3} \mathrm{O}_{5}$. The XRPD pattern at $300 \mathrm{~K}$ under atmospheric pressure $(P=0.1 \mathrm{MPa})$ is shown in Fig. 1d and Supplementary Fig. 2. Rietveld analysis indicates that this sample is composed of $80.0(2) \% \quad \lambda-\mathrm{Ti}_{3} \mathrm{O}_{5} \quad$ and $20.0(2) \% \quad \beta-\mathrm{Ti}_{3} \mathrm{O}_{5} . \quad \lambda-\mathrm{Ti}_{3} \mathrm{O}_{5}$ adopts a monoclinic crystal structure (space group $\mathrm{C} 2 / \mathrm{m}$ ) with lattice parameters of $a=9.83119(19) \AA, \quad b=3.78798(7) \AA$, $c=9.97039(19) \AA$ and $\beta=91.2909(7)$, and a unit cell volume, $V=371.207(12) \AA^{3} . \lambda-\mathrm{Ti}_{3} \mathrm{O}_{5}$ has three symmetry-inequivalent $\mathrm{Ti}$ sites, $\operatorname{Ti}(1), \operatorname{Ti}(2)$ and $\mathrm{Ti}(3)$, and five-symmetry-inequivalent $\mathrm{O}$ sites, $\mathrm{O}(1)$ to $\mathrm{O}(5)$. All the $\mathrm{Ti}$ sites form a six-coordinate structure. In the previous investigation ${ }^{6}$ of the same polymorph prepared from anatase- $\mathrm{TiO}_{2}$ nanoparticles, we observed some indications of a pressure effect. In the present research, the sample was pressed at various external pressures with a pellet press, and XRPD patterns were measured for the pellets after pressure release. With increasing $P$, the intensity of the XRPD peaks of $\lambda-\mathrm{Ti}_{3} \mathrm{O}_{5}$ decreased and those of $\beta-\mathrm{Ti}_{3} \mathrm{O}_{5}$ increased (Fig. $1 \mathrm{~d}$ and Supplementary Fig. 3). The pressure where the fraction of $\lambda-\mathrm{Ti}_{3} \mathrm{O}_{5}$ becomes $50 \%\left(P_{1 / 2}\right)$ is $\sim 60 \mathrm{MPa}$ as shown in Fig. 1e. The crystal structure of $\beta-\mathrm{Ti}_{3} \mathrm{O}_{5}$ is monoclinic (space group $C 2 / m ; \quad a=9.75252(18) \AA, \quad b=3.80034(6) \AA$, $c=9.44413(19) \AA, \quad \beta=91.5322(10)^{\circ} \quad$ and $\left.\quad V=349.902(11) \AA^{3}\right)$ (Supplementary Fig. 4). After pressurizing the sample and releasing the pressure at room temperature, heating the sample causes $\beta-\mathrm{Ti}_{3} \mathrm{O}_{5}$ to revert back to $\lambda-\mathrm{Ti}_{3} \mathrm{O}_{5}$ at $470 \mathrm{~K}$ (Fig. $1 \mathrm{~d}$,f and Supplementary Fig. 5a). Above $530 \mathrm{~K}, \lambda-\mathrm{Ti}_{3} \mathrm{O}_{5}$ further transforms to $\alpha-\mathrm{Ti}_{3} \mathrm{O}_{5}$. On the other hand, in the cooling process from 620 to $300 \mathrm{~K}, \alpha-\mathrm{Ti}_{3} \mathrm{O}_{5}$ returns to $\lambda-\mathrm{Ti}_{3} \mathrm{O}_{5}$ (Supplementary Fig. 5b). This $\lambda-\mathrm{Ti}_{3} \mathrm{O}_{5}$ is very stable in the wide temperature range of $0<T<530 \mathrm{~K}$. Furthermore, when external pressure was applied to this recovered $\lambda-\mathrm{Ti}_{3} \mathrm{O}_{5}$ sample, $\lambda-\mathrm{Ti}_{3} \mathrm{O}_{5}$ exhibited again the phase transition to $\beta-\mathrm{Ti}_{3} \mathrm{O}_{5}$ (Supplementary Figs 6 and 7).

The visualized electron density distributions of $\lambda-\mathrm{Ti}_{3} \mathrm{O}_{5}$ and $\beta-\mathrm{Ti}_{3} \mathrm{O}_{5}$ obtained using MEM from the XRPD patterns, are shown in Fig. 2a. The MEM image of $\lambda-\mathrm{Ti}_{3} \mathrm{O}_{5}$ shows that the electron density is spread between both $\mathrm{Ti}$ and $\mathrm{O}$ atoms, while in $\beta-\mathrm{Ti}_{3} \mathrm{O}_{5}$, the electron density is localized around each atom. This result indicates the electron delocalized character of $\lambda-\mathrm{Ti}_{3} \mathrm{O}_{5}$ and localized character of $\beta-\mathrm{Ti}_{3} \mathrm{O}_{5}$, which are consistent with the fact that $\lambda-\mathrm{Ti}_{3} \mathrm{O}_{5}$ is a metallic conductor and $\beta-\mathrm{Ti}_{3} \mathrm{O}_{5}$ is a semiconductor. In addition, the visualized electron density distribution of $\lambda-\mathrm{Ti}_{3} \mathrm{O}_{5}$ in the $b c$ plane well reproduces the HRTEM image, as mentioned in Figs $1 b$ and $c$.

First-principles calculation of phonon mode. To elucidate the pressure-induced phase transition, first-principles phonon mode calculations were conducted. Figure $2 \mathrm{~b}$ shows the phonon density of states (DOS) based on the lattice vibrations for $\lambda-\mathrm{Ti}_{3} \mathrm{O}_{5}$ and $\beta$ - 
a

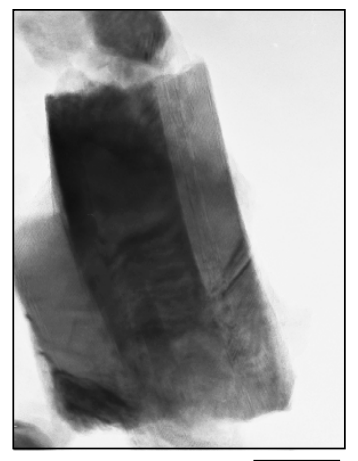

b

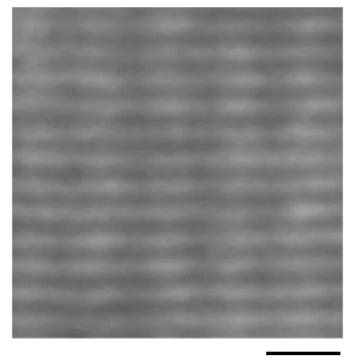

C

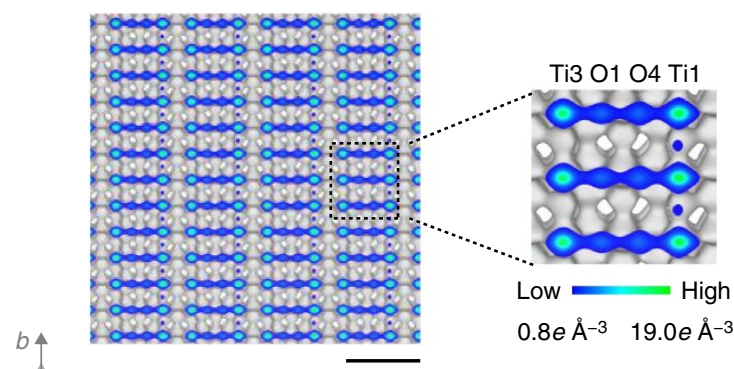

d



e
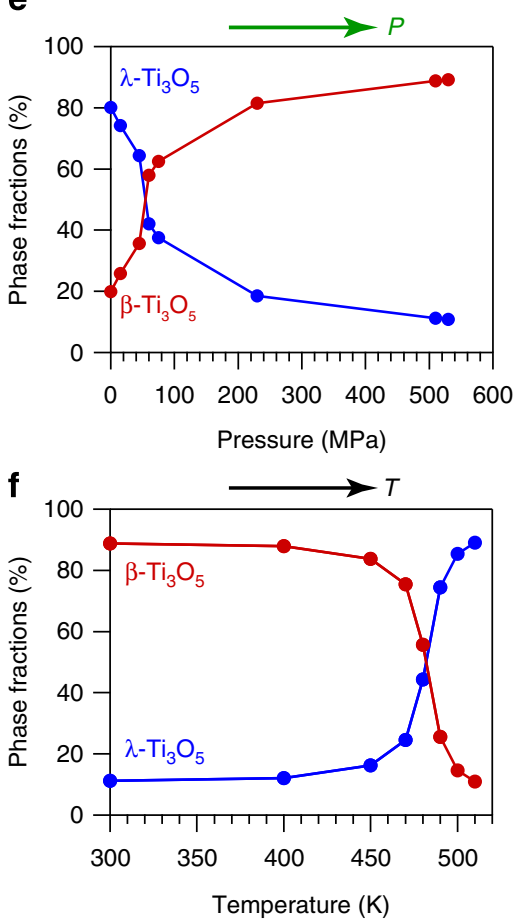

Figure 1 | Morphology of stripe-type- $\lambda-\mathrm{Ti}_{3} \mathrm{O}_{5}$ and pressure-and-heat-induced phase transition between $\lambda-\mathrm{Ti}_{3} \mathrm{O}_{5}$ and $\boldsymbol{\beta}-\mathrm{Ti}_{3} \mathrm{O}_{5}$. (a) $\mathrm{TEM}$ image of stripetype- $\lambda-\mathrm{Ti}_{3} \mathrm{O}_{5}$. The scale bar below the TEM image indicates $50 \mathrm{~nm}$. (b) HRTEM image of the surface of stripe-type- $\lambda$ - $\mathrm{Ti}_{3} \mathrm{O}_{5}$ showing the atomic arrangement on the $b c$ plane. The scale bar below the TEM image indicates $1 \mathrm{~nm}$. (c) Visualized electron density maps on the bc plane of stripe-type- $\lambda$ $\mathrm{Ti}_{3} \mathrm{O}_{5}$ obtained by the MEM (isosurface $0.8 \mathrm{e} \AA^{-3}$ ). The scale bar below the electron density map (left) indicates $1 \mathrm{~nm}$. (d) Pressure $(P)$ and temperature ( $T$ ) dependence of the XRPD patterns $(\lambda=1.5418 \AA$ ). The ambient-temperature XRPD pattern of the as-prepared sample at atmospheric pressure $(P=0.1 \mathrm{MPa})$ is shown in the front, followed by XRPD patterns of the pellet samples pressurized by $P=15-530 \mathrm{MPa}$, measured after pressure release. These are followed by the XRPD patterns of pressure-produced $\beta-\mathrm{Ti}_{3} \mathrm{O}_{5}$ with increasing temperature from $300 \mathrm{~K}$ to $510 \mathrm{~K}$. (e) Pressure evolution of the phase fractions of $\lambda-\mathrm{Ti}_{3} \mathrm{O}_{5}$ (blue) and $\beta-\mathrm{Ti}_{3} \mathrm{O}_{5}$ (red). The pressure where the fraction of $\lambda-\mathrm{Ti}_{3} \mathrm{O}_{5}$ becomes $50 \%\left(P_{1 / 2}\right)$ is an extremely small value of $\sim 60$ MPa. (f) Temperature evolution of the phase fractions of $\lambda-\mathrm{Ti}_{3} \mathrm{O}_{5}$ (blue) and $\beta-\mathrm{Ti}_{3} \mathrm{O}_{5}$ (red) in the heating process.

$\mathrm{Ti}_{3} \mathrm{O}_{5}$. The phonon dispersion and phonon frequencies at the Brillouin zone centre, $\Gamma$ point, for each of the phonon dispersions are listed in Supplementary Fig. 8. Comparison of the two crystal structures shows that the coordination geometry of $\mathrm{Ti}(3)$ is different between $\lambda-\mathrm{Ti}_{3} \mathrm{O}_{5}$ and $\beta-\mathrm{Ti}_{3} \mathrm{O}_{5} ; \mathrm{Ti}(3)$ is connected to $\mathrm{O}(5)$ in $\lambda-\mathrm{Ti}_{3} \mathrm{O}_{5}$, while it bonds to $\mathrm{O}(4)$ in $\beta-\mathrm{Ti}_{3} \mathrm{O}_{5}$. Therefore, in the pressure-induced phase transition from $\lambda-\mathrm{Ti}_{3} \mathrm{O}_{5}$ to $\beta-\mathrm{Ti}_{3} \mathrm{O}_{5}$, the $\mathrm{Ti}(3)-\mathrm{O}(5)$ bond is considered to break, and the $\mathrm{Ti}(3)-\mathrm{O}(4)$ bond to form. The corresponding phonon modes of $\lambda-\mathrm{Ti}_{3} \mathrm{O}_{5}$ lie at 248.6, 318.5 and $445.8 \mathrm{~cm}^{-1}$. For example, for the $\mathrm{B}_{\mathrm{u}}$ phonon mode at $445.8 \mathrm{~cm}^{-1}, \mathrm{Ti}(3)$ vibrates significantly toward $\mathrm{O}(4)$ and moves further away from $\mathrm{O}(5)$ (Fig. 2c (upper) and
Supplementary Movie 1). On the contrary, in the course of the thermal phase transition (that is, heat-storage process) from $\beta-\mathrm{Ti}_{3} \mathrm{O}_{5}$ to $\lambda-\mathrm{Ti}_{3} \mathrm{O}_{5}$, the $\mathrm{Ti}(3)-\mathrm{O}(4)$ bond is broken and the $\mathrm{Ti}(3)-\mathrm{O}(5)$ bond is generated. The corresponding phonon modes now lie at 226.7 and $339.3 \mathrm{~cm}^{-1}$. For example, visualization of the $B_{u}$ phonon mode at $226.7 \mathrm{~cm}^{-1}$ shows that $\operatorname{Ti}(3)$ significantly vibrates towards $\mathrm{O}(5)$ (Fig. 2c (lower) and Supplementary Movie 2).

Accumulated heat energy and pressure-released energy. To investigate the heat-storage process from pressure-produced $\beta-\mathrm{Ti}_{3} \mathrm{O}_{5}$ to $\lambda-\mathrm{Ti}_{3} \mathrm{O}_{5}$ and the amount of accumulated thermal 
a
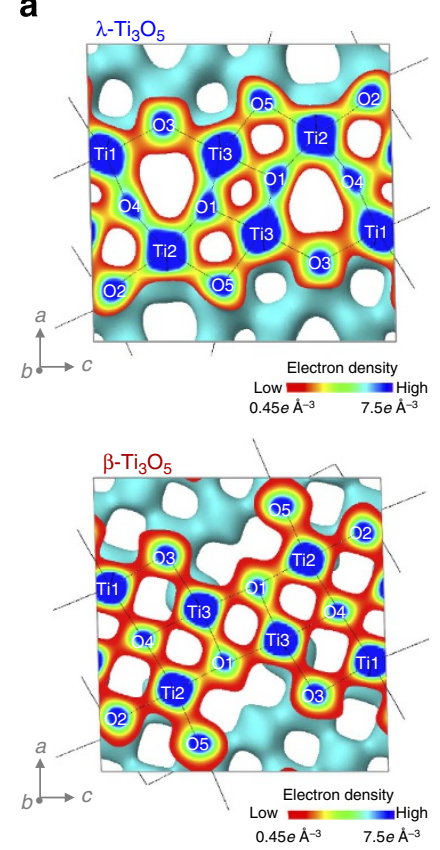

b
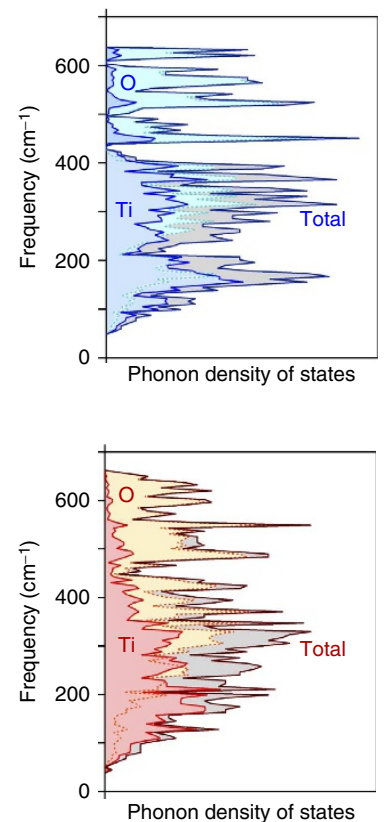

C
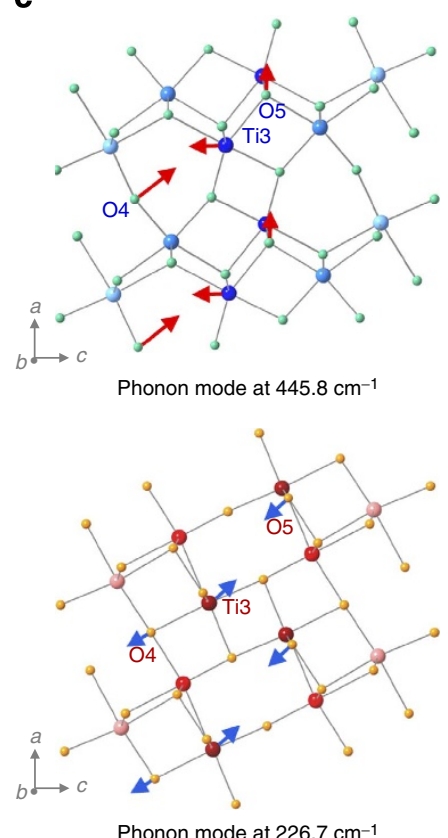

Figure 2 | Electron density maps and the phonon modes of $\boldsymbol{\lambda}-\mathbf{T i}_{\mathbf{3}} \mathbf{O}_{\mathbf{5}}$ and $\mathbf{\beta}-\mathbf{T i}_{\mathbf{3}} \mathbf{O}_{\mathbf{5}}$. (a) Visualized electron density maps (isosurface $0.45 e \AA-3$ ) of $\boldsymbol{\lambda}-\mathrm{Ti}_{3} \mathrm{O}_{5}$ (upper) and $\beta-\mathrm{Ti}_{3} \mathrm{O}_{5}$ (lower) obtained using MEM from the XRPD patterns. (b) Phonon density of state (DOS) for $\lambda$ - $\mathrm{Ti}_{3} \mathrm{O}_{5}$ (upper) and $\beta$-Ti $\mathrm{O}_{3} \mathrm{O}_{5}$ (lower). Blue, light blue and grey areas indicate the contributions from phonons due to $\mathrm{Ti}, \mathrm{O}$, and the total phonon $\mathrm{DOS}$, respectively for $\lambda$ - $\mathrm{Ti}_{3} \mathrm{O}_{5}$ (upper). Red, orange and grey areas indicate the contributions from phonons due to Ti, $\mathrm{O}$ and the total phonon DOS, respectively, for $\beta$-Ti $i_{3} \mathrm{O}_{5}$ (lower). (c) Schematic illustration of the $\mathrm{B}_{u}$ phonon mode at $445.8 \mathrm{~cm}^{-1}$ for $\lambda-\mathrm{Ti}_{3} \mathrm{O}_{5}$ (upper) and the $\mathrm{B}_{\mathrm{u}}$ phonon mode at $226.7 \mathrm{~cm}^{-1}$ for $\beta-\mathrm{Ti}_{3} \mathrm{O}_{5}$ (lower). Arrows and their lengths indicate the direction of the movement of the atoms and the relative amplitude of oscillation, respectively (see Supplementary Movies 1 and 2).

energy in the system, heat capacity measurements were performed. First, we investigated the heat capacity of the pressure-produced $\beta-\mathrm{Ti}_{3} \mathrm{O}_{5}$. In the temperature region from 5 to $300 \mathrm{~K}$, specific heat was measured by the relaxation technique using the physical properties measurement system (Fig. 3a), and above $300 \mathrm{~K}$, specific heat accompanying the thermal phase transition from pressure-produced $\beta-\mathrm{Ti}_{3} \mathrm{O}_{5}$ to $\lambda-\mathrm{Ti}_{3} \mathrm{O}_{5}$ was measured by differential scanning calorimetry (DSC; Fig. $3 \mathrm{~b}$ ). By combining the results from the physical properties measurement system and DSC measurements and integrating with temperature, the experimental enthalpy $(H)$ curves of $\lambda-\mathrm{Ti}_{3} \mathrm{O}_{5}$ and $\beta-\mathrm{Ti}_{3} \mathrm{O}_{5}$ versus temperature were obtained up to $600 \mathrm{~K}$ (Fig. $3 \mathrm{c}$; see Methods). The transition enthalpy $(\Delta H)$ associated with the firstorder phase transition from $\beta-\mathrm{Ti}_{3} \mathrm{O}_{5}$ to $\lambda-\mathrm{Ti}_{3} \mathrm{O}_{5}$ was $230 \pm 20 \mathrm{~kJ}$ $\mathrm{L}^{-1}\left(12 \pm 1 \mathrm{~kJ} \mathrm{~mol}^{-1}\right)$. In the temperature decreasing process of the DSC measurement, there was no peak, indicating that the accumulated heat energy of the phase transition from $\beta-\mathrm{Ti}_{3} \mathrm{O}_{5}$ to $\lambda-\mathrm{Ti}_{3} \mathrm{O}_{5}$ was conserved in the system.

Next the released energy of the pressure-induced phase transition from stripe-type- $\lambda-\mathrm{Ti}_{3} \mathrm{O}_{5}$ to $\beta-\mathrm{Ti}_{3} \mathrm{O}_{5}$ was measured using a high-pressure micro-DSC measurement system at room temperature. After applying pressure, heat energy of $240 \pm 40 \mathrm{~kJ} \mathrm{~L}^{-1}$ was released, which almost corresponds to the heat accumulated energy (Fig. 3d). Therefore, this material conserves the heat energy of the phase transition from pressureproduced $\beta-\mathrm{Ti}_{3} \mathrm{O}_{5}$ to $\lambda-\mathrm{Ti}_{3} \mathrm{O}_{5}$ and releases the accumulated heat energy by applying low pressure through the pressureinduced phase transition from $\lambda-\mathrm{Ti}_{3} \mathrm{O}_{5}$ to $\beta-\mathrm{Ti}_{3} \mathrm{O}_{5}$ (Supplementary Movie 3).

Thermal conductivity and sensible heat-storage performance. Bricks and concrete are useful as sensible heat-storage materials ${ }^{20,26-28}$ since they release thermal energy slowly. Thermal conductivity measurements were performed for the stripe-type- $\lambda-\mathrm{Ti}_{3} \mathrm{O}_{5}$ and pressure-produced $\beta-\mathrm{Ti}_{3} \mathrm{O}_{5}$. The thermal conducti-

vities were $0.20 \pm 0.02 \mathrm{~W} \mathrm{~m}^{-1} \mathrm{~K}^{-1}$ and $0.41 \pm 0.02 \mathrm{~W} \mathrm{~m}^{-1} \mathrm{~K}^{-1}$ for $\lambda-\mathrm{Ti}_{3} \mathrm{O}_{5}$ and $\beta-\mathrm{Ti}_{3} \mathrm{O}_{5}$, respectively, which are similar to the values of bricks (for example, $0.16 \mathrm{~W} \mathrm{~m}^{-1} \mathrm{~K}^{-1}$ ) ${ }^{26}$ and concrete (for example, $\left.0.57 \mathrm{~W} \mathrm{~m}^{-1} \mathrm{~K}^{-1}\right)^{28}$.

Current-induced and light-induced phase transitions. Electric current was flowed to the pressure-produced $\beta-\mathrm{Ti}_{3} \mathrm{O}_{5}$ sample at $298 \mathrm{~K}$. By flowing a current of $0.4 \mathrm{~A} \mathrm{~mm}^{-2}$, the colour of the sample changed from brown to dark blue (Fig. 4a). The XRPD patterns before and after flowing the current indicate that $\beta-\mathrm{Ti}_{3} \mathrm{O}_{5}$ is transformed into $\lambda-\mathrm{Ti}_{3} \mathrm{O}_{5}$ (Fig. $4 \mathrm{~b}$ and Supplementary Movie 4). The electric current dependence on the conversion from the pressure-produced $\beta-\mathrm{Ti}_{3} \mathrm{O}_{5}$ to $\lambda-\mathrm{Ti}_{3} \mathrm{O}_{5}$ shows that the threshold current value of the current-induced phase transition is $0.2 \mathrm{~A} \mathrm{~mm}^{-2}$ (Supplementary Fig. 9). The origin of this current-induced phase transition is regarded as breaking of charge ordering or (and) Joule heat ${ }^{16-18}$. The mechanism by breaking of charge ordering is considered as follows: $\beta-\mathrm{Ti}_{3} \mathrm{O}_{5}$ is a charge-localized state whose charge is localized on $\mathrm{Ti}^{3+}(3)$ with empty orbital on $\mathrm{Ti}^{4+}(2)$. In contrast, $\lambda-\mathrm{Ti}_{3} \mathrm{O}_{5}$ is a chargedelocalized state whose charge is delocalized on $\operatorname{Ti}(2)$ and $\operatorname{Ti}(3)$. By flowing electric current to $\beta-\mathrm{Ti}_{3} \mathrm{O}_{5}$, the localized charge on $\mathrm{Ti}(3)$ is forcedly moved to the empty orbital on of $\mathrm{Ti}(2)$, resulting in a transition to metallic $\lambda-\mathrm{Ti}_{3} \mathrm{O}_{5}$.

Light irradiation experiment was also conducted on a pressureproduced $\beta-\mathrm{Ti}_{3} \mathrm{O}_{5}$. The reverse phase transition from $\beta-\mathrm{Ti}_{3} \mathrm{O}_{5}$ to $\lambda-\mathrm{Ti}_{3} \mathrm{O}_{5}$ was observed by irradiation of $410-\mathrm{nm}$ laser light (Supplementary Fig. 10 and Supplementary Movie 5). 
a

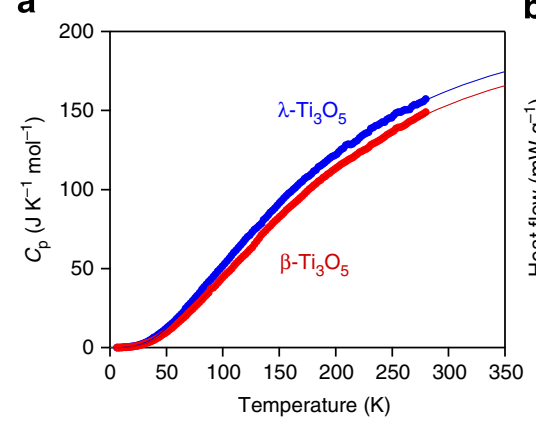

b

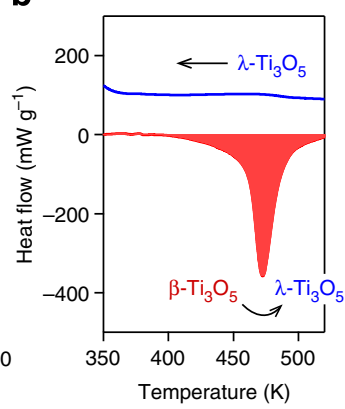

C



Figure 3 | Thermodynamic properties of stripe-type- $\lambda-\mathrm{Ti}_{3} \mathrm{O}_{5}$ and pressure-produced $\boldsymbol{\beta}-\mathrm{Ti}_{3} \mathbf{O}_{\mathbf{5}}$. (a) Molar heat capacity of $\lambda-\mathrm{Ti}_{3} \mathrm{O}_{5}$ (blue) and $\beta-\mathrm{Ti}_{3} \mathrm{O}_{5}$ (red) as a function of temperature. Experimental data were fitted with a Debye model (see Methods). (b) DSC charts of the pressureproduced $\beta-\mathrm{Ti}_{3} \mathrm{O}_{5}$ with increasing temperature and $\lambda-\mathrm{Ti}_{3} \mathrm{O}_{5}$ with decreasing temperature. $A$ peak due to the latent heat of the first-order phase transition from $\beta-\mathrm{Ti}_{3} \mathrm{O}_{5}$ to $\lambda-\mathrm{Ti}_{3} \mathrm{O}_{5}\left(230 \mathrm{~kJ} \mathrm{~L}{ }^{-1}\right)$ was observed in the heating process, whereas no peak was observed in the cooling process. (c) Temperature dependence of the enthalpy $(H)$ for $\lambda-\mathrm{Ti}_{3} \mathrm{O}_{5}$ (blue) and $\beta$ $\mathrm{Ti}_{3} \mathrm{O}_{5}$ (red). When pressure is applied to $\lambda-\mathrm{Ti}_{3} \mathrm{O}_{5}$, the accumulated heat energy is released as shown in the lower enlarged figure (see Supplementary Movie 3). (d) Pressure-released heat energy accompanying the pressure-induced phase transition from stripe-type- $\lambda-\mathrm{Ti}_{3} \mathrm{O}_{5}$ to $\beta-\mathrm{Ti}_{3} \mathrm{O}_{5}$. Pressure was applied at $t=0$.

\section{Discussion}

The generation of stripe-type- $\lambda-\mathrm{Ti}_{3} \mathrm{O}_{5}$ originates from the change in the Gibbs free energy $(G)$ of the material compared with the bulk or single crystal $\mathrm{Ti}_{3} \mathrm{O}_{5}$. This change in the $G$ value is considered to be due to the interface (and/or surface) energy of the nanoscale domain. It is noted that there is no oxygen vacancy, which was confirmed by electron spin resonance. To understand why the stripe-type- $\lambda-\mathrm{Ti}_{3} \mathrm{O}_{5}$ undergoes a pressure-induced phase transition to $\beta-\mathrm{Ti}_{3} \mathrm{O}_{5}$, we considered the thermodynamics of the present phase transition phenomena using the mean-field model, developed by Slichter and Drickamer ${ }^{29}$. In this model, $G$ is a


b
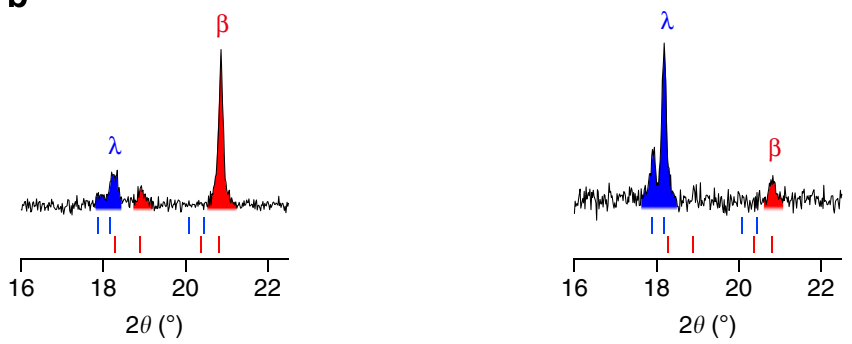

Figure 4 | Current-induced phase transition from $\beta-\mathrm{Ti}_{3} \mathrm{O}_{5}$ to $\lambda-\mathrm{Ti}_{3} \mathrm{O}_{5}$. An electric current of $0.4 \mathrm{~A} \mathrm{~mm}^{-2}$ flowed through the pressure-produced $\beta-\mathrm{Ti}_{3} \mathrm{O}_{5}$ at $298 \mathrm{~K}$. (a) Photographs of the pressure-produced $\beta-\mathrm{Ti}_{3} \mathrm{O}_{5}$ before (left) and after the application of an electric current of $0.4 \mathrm{~A} \mathrm{~mm}^{-2}$ (right). (b) XRPD pattern in the $2 \theta$ range of $16.0-22.5^{\circ}$ of the pressure-produced $\beta-\mathrm{T}_{3} \mathrm{O}_{5}$ (left) and after the application of the electric current (right). Blue and red areas mark the peaks of $\lambda-\mathrm{Ti}_{3} \mathrm{O}_{5}$ and $\beta-\mathrm{Ti}_{3} \mathrm{O}_{5}$, respectively.

described by $\Delta H$, the transition entropy $(\Delta S)$ and the interaction parameter between $\lambda-\mathrm{Ti}_{3} \mathrm{O}_{5}$ and $\beta-\mathrm{Ti}_{3} \mathrm{O}_{5}$ phases. The calculation shows that at atmospheric pressure $(P=0.1 \mathrm{MPa})$, the sample exists as $\lambda-\mathrm{Ti}_{3} \mathrm{O}_{5}$ (Supplementary Movie 6). This is because $\lambda-\mathrm{Ti}_{3} \mathrm{O}_{5}$ is synthesized by sintering at a high temperature, and it remains as $\lambda-\mathrm{Ti}_{3} \mathrm{O}_{5}$ with decreasing temperature due to the energy barrier between $\lambda-\mathrm{Ti}_{3} \mathrm{O}_{5}$ and $\beta-\mathrm{Ti}_{3} \mathrm{O}_{5}$ as shown in the $G$ versus fraction $(x)$ of $\lambda-\mathrm{Ti}_{3} \mathrm{O}_{5}$ curves (Fig. $5 \mathrm{a}$ (i)). On the contrary, on applying external pressure, the $G$ versus $x$ curves change; for example, the energy barrier disappears $<400 \mathrm{~K}$ when $P$ is $60 \mathrm{MPa}$, and hence, $\lambda-\mathrm{Ti}_{3} \mathrm{O}_{5}$ transforms into $\beta-\mathrm{Ti}_{3} \mathrm{O}_{5}$ on applying pressure (Fig. 5a (ii)). The $x$ versus temperature curves of $P=0.1 \mathrm{MPa}$ and $P=60 \mathrm{MPa}$ are shown in Fig. 5b. As shown in Fig. 5c, $x$ versus pressure plots indicate the threshold of the pressure-induced phase transition. The origin of the pressureinduced phase transition is the $P \Delta V$ term of $\Delta H(=\Delta U+P \Delta V)$, where $\Delta U$ and $\Delta V$ are the changes of internal energy and volume, respectively. At such a low pressure, the pressure-induced change on $\Delta U$ is very small and negligible. In fact, the phonon mode calculation under external pressure shows that the pressureinduced change of $\Delta U$ is $\sim 1 \times 10^{-3} \mathrm{~kJ} \mathrm{~mol}^{-1}$ at $60 \mathrm{MPa}$, which is two orders smaller compared with $P \Delta V=0.19 \mathrm{~kJ} \mathrm{~mol}^{-1}$. The pressure-induced change on $\Delta S$ is also very small and cannot contribute to the pressure-induced phase transition in the present system (see Methods, Supplementary Fig. 11 and Supplementary Tables 1,2$)$. It is noted that the observed $x$ versus $P$ plots of Fig. 1e is somewhat gradual. This is explained by the presence of a distribution in the transition pressure of the Slichter and Drickamer model, which may be due to the crystal size distribution. We have simulated this gradual pressure-induced phase transition with a distribution of transition pressures (Supplementary Fig. 12).

In summary, we report the first metal oxide capable of conserving the accumulated heat energy of a phase transition. Stripe-type- $\lambda-\mathrm{Ti}_{3} \mathrm{O}_{5}$ can store a large heat energy of $230 \mathrm{~kJ} \mathrm{~L}^{-1}$, and this energy can be released by applying external pressure only when demanded. The magnitude of the required pressure is extremely small, $\sim 60 \mathrm{MPa}$. This value is remarkably smaller than the typical pressures observed in the pressure-induced phase 
a

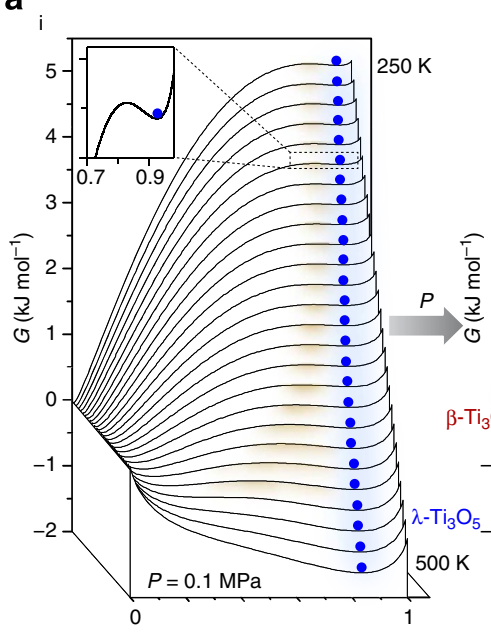

Fraction $(x)$

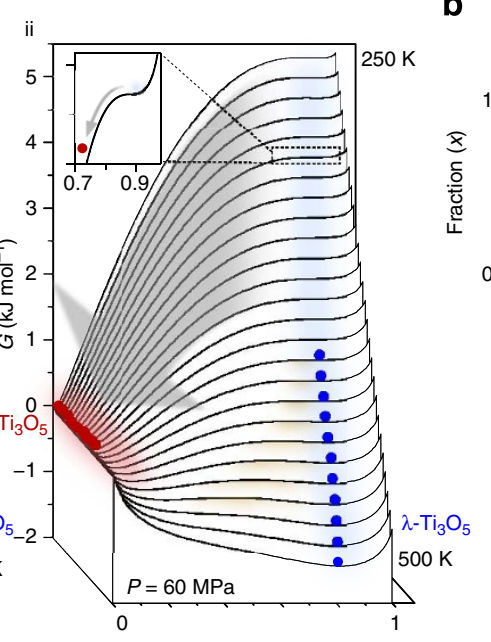

Fraction $(x)$ b

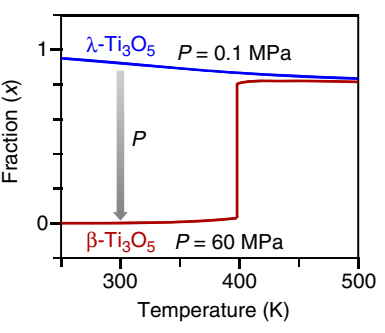

C



Figure 5 | Mechanism of the pressure-induced phase transition based on a thermodynamic model. (a) Gibbs free energy $(G)$ versus $\lambda$-Ti $i_{3} \mathrm{O}_{5}$ fraction $(x)$ for every $10 \mathrm{~K}$ between $250 \mathrm{~K}$ to $500 \mathrm{~K}$ calculated using the Slichter-Drickamer mean-field model at $P=0.1 \mathrm{MPa}$ (i) and $60 \mathrm{MPa}$ (ii). Blue and red circles indicate $\lambda-\mathrm{Ti}_{3} \mathrm{O}_{5}$ and $\beta-\mathrm{Ti}_{3} \mathrm{O}_{5}$, respectively. $\lambda-\mathrm{Ti}_{3} \mathrm{O}_{5}$ undergoes a pressure-induced phase transition to $\beta-\mathrm{Ti}_{3} \mathrm{O}_{5}$ because the energy barrier (shown by brown shadows) disappears by the application of external pressure above $\sim 60 \mathrm{MPa}$ as shown in the insets (see Supplementary Movie 6). (b) Calculated $x$ versus temperature curves at $P=0.1 \mathrm{MPa}$ (blue) and $60 \mathrm{MPa}$ (red). (c) Calculated $x$ versus pressure curve at $300 \mathrm{~K}$ indicating a threshold pressure of $\sim 60 \mathrm{MPa}$.

transitions in metal oxide materials ${ }^{30-35}$ and metallic compounds $^{36-41}$, for example, the pressure-induced phase transition from rutile- $\mathrm{TiO}_{2}$ to baddeleyite-type $\mathrm{TiO}_{2}$ at $1,043 \mathrm{~K}$ occurs at $20,000 \mathrm{MPa}(=20 \mathrm{GPa})^{30}$. From the viewpoint of the energy balance of the thermodynamic cycle, pressure of $60 \mathrm{MPa}$ corresponds to $\sim 10 \mathrm{~kJ} \mathrm{~L}^{-1}$, which is $<5 \%$ of the pressurereleasing heat energy. Pressure of $\sim 60 \mathrm{MPa}$ can be realized even by the water pressure of a high-pressure washing machine, and hence, $\lambda-\mathrm{Ti}_{3} \mathrm{O}_{5}$ has the potential to be employed as pressuresensitive sheets or reusable portable heating pads. In addition, since $\lambda-\mathrm{Ti}_{3} \mathrm{O}_{5}$ is a metallic conductor and $\beta-\mathrm{Ti}_{3} \mathrm{O}_{5}$ is a semiconductor, it has possibilities as a pressure-sensitive conductivity sensor or pressure-sensitive optical sensor. Furthermore, because $\lambda-\mathrm{Ti}_{3} \mathrm{O}_{5}$ is composed of common elements (titanium and oxygen), it is safe and environmentally friendly. $\lambda-\mathrm{Ti}_{3} \mathrm{O}_{5}$ could be useful for heat-retaining systems for residential use and may realize more efficient uses of industrial waste heat generated from furnaces (Supplementary Fig. 13) ${ }^{42,43}$. In addition, light-induced and current-induced phase transitions from pressure-produced $\beta-\mathrm{Ti}_{3} \mathrm{O}_{5}$ to $\lambda-\mathrm{Ti}_{3} \mathrm{O}_{5}$ are also observed, that is, stripe-type- $\lambda-\mathrm{Ti}_{3} \mathrm{O}_{5}$ shows reversible pressure-and-light-induced phase transition and reversible pressure-and-current-induced phase transition. These effects are also attractive phenomena from the viewpoint of advanced electronic devices.

\footnotetext{
Methods

Material. A new series of $\lambda-\mathrm{Ti}_{3} \mathrm{O}_{5}$ nanocrystallites was produced by sintering rutile- $\mathrm{TiO}_{2}$ particles in a hydrogen atmosphere (flow rates of $0.7 \mathrm{dm}^{3} \mathrm{~min}^{-1}$ ) at $1,117^{\circ} \mathrm{C}$ for $2 \mathrm{~h}$, followed by a slow cooling process of $\sim 9 \mathrm{~h}$ from the sintering temperature to room temperature (Supplementary Fig. 14). Elemental analysis using inductively coupled plasma mass spectrometry confirms that the formula is $\mathrm{Ti}_{3.00(1)} \mathrm{O}_{5.00(6)}$; Calc.: Ti, 64.2\%. Found: $\mathrm{Ti}, 64.2(1) \%$. The experimentally obtained density is $4.000 \pm 0.048 \mathrm{~g} \mathrm{~cm}^{-3}$, which is consistent with the theoretical value of $4.00 \mathrm{~g} \mathrm{~cm}^{-3}$ from the crystal structure of $\lambda-\mathrm{Ti}_{3} \mathrm{O}_{5}$ as determined by XRPD measurements. SEM and TEM images of the obtained sample show a coral-like morphology with particle size of $\sim 4 \times 1 \mu \mathrm{m}$, composed of rectangular-shaped nanorods, of which the majority are $\sim 200 \times 30 \mathrm{~nm}$ dimensions (Supplementary Fig. 1a). The Fourier transform analysis of the HRTEM image showed that the growth direction of the nanorods is along the crystallographic $b$ axis. This new series of $\lambda-\mathrm{Ti}_{3} \mathrm{O}_{5}$ have larger crystal size than the previous series, which were prepared from anatase- $\mathrm{TiO}_{2}$ (ref. 6; Supplementary Fig. 1b).
}

XRPD measurements. XRPD measurements were performed with a Rigaku Ultima IV diffractometer with $\mathrm{Cu} K_{\alpha}$ radiation $(\lambda=1.5418 \AA)$. The temperaturedependent XRPD measurements were undertaken using a high-temperature chamber with atmosphere control (RIGAKU-OAT003S) under $\mathrm{N}_{2}$ flow. The RIETAN-FP computer programme was used for the Rietveld analyses, while Dysnomia was used for the MEM analyses. The refined crystal structures and charge densities were visualized by the computer programme VESTA. Although both $\lambda-\mathrm{Ti}_{3} \mathrm{O}_{5}{ }^{6}$ and its high-temperature phase ${ }^{44,45}$ can be considered as candidates of the present material with $C 2 / m$ crystal structure, we assigned the present material to $\lambda-\mathrm{Ti}_{3} \mathrm{O}_{5}$ because it is obtained by a very slow cooling process taking of ca. $9 \mathrm{~h}$ from the sintering temperature to room temperature, and it is thermally stable.

Heat capacity measurements. To investigate the temperature dependence of the lattice specific heat, $C(T)$, in the temperature range of $5-300 \mathrm{~K}$, we carried out curve fitting of the observed plots with the equation based on the two-Debye model ${ }^{46}$ expressed by $C(T)=\sum_{i=1}^{2} 9 R c_{i}\left(T / \theta_{i}\right)^{3} \int_{0}^{\theta_{i} / T} x^{4} e^{x} /\left(e^{x}-1\right)^{2} \mathrm{~d} x$, where $R$ is gas constant, $c_{i}$ is coefficient, $\theta_{i}$ is Debye temperature, $x$ is $\hbar \omega / k_{\mathrm{B}} T$, $\hbar$ is the reduced Planck constant, $\omega$ is phonon frequency and $k_{\mathrm{B}}$ is Boltzmann constant, with the fit parameters of $c_{1}=3.2(1), c_{2}=5.6(1), \theta_{1}=4.1(1) \times 10^{2} \mathrm{~K}$ and $\theta_{2}=9.3(1) \times 10^{2} \mathrm{~K}$ for $\lambda-\mathrm{Ti}_{3} \mathrm{O}_{5}$, and $c_{1}=2.7(1), c_{2}=5.8(1), \theta_{1}=4.3(1) \times 10^{2} \mathrm{~K}$ and $\theta_{2}=9.3(2) \times 10^{2} \mathrm{~K}$ for $\beta-\mathrm{Ti}_{3} \mathrm{O}_{5}$. We then developed the temperature dependence curve of the specific heat in the temperature range of 5-600 K using both the fitted curve and the anomalous specific heat associated with the first-order phase transition from $\beta-\mathrm{Ti}_{3} \mathrm{O}_{5}$ to $\lambda-\mathrm{Ti}_{3} \mathrm{O}_{5}$ obtained from the DSC measurement.

Released heat energy on pressure application. Released heat energy on pressure application was measured with a high-pressure DSC measurement system ( $\mu$ DSC VII, SETARAM Instrumentation) at $300 \mathrm{~K}$. Pressure application of $40 \mathrm{MPa}$ was achieved by instant injection of $\mathrm{N}_{2}$ gas into the sample cell.

Thermal conductivity measurements. The specific heat and thermal diffusivity of $\lambda-\mathrm{Ti}_{3} \mathrm{O}_{5}$ and $\beta-\mathrm{Ti}_{3} \mathrm{O}_{5}$ pellet samples were measured with a DSC measurement system (DSC200F3 Maia (NETZSCH), NSST Co., Ltd.) and Light Flash Apparatus (LFA447NanoFlash, NSST Co., Ltd.), respectively.

First-principles phonon mode calculations. First-principles calculations based on the density functional theory were carried out for $\lambda-\mathrm{Ti}_{3} \mathrm{O}_{5}$ and $\beta-\mathrm{Ti}_{3} \mathrm{O}_{5}$ using the VASP (Vienna $a b$ initio simulation package) code. The wavefunctions based on plane waves and potentials of the core orbitals were represented by the projectoraugmented wave of Blöchl, and the exchange-correlation term was evaluated by the generalized gradient approximation by Perdew, Burke, and Ernzerhof. The crysta structures of $\lambda-\mathrm{Ti}_{3} \mathrm{O}_{5}$ and $\beta-\mathrm{Ti}_{3} \mathrm{O}_{5}$ obtained from the XRPD measurements were used for computed models as the initial structures. The lattice parameters and atomic positions were optimized under no pressure and $1000 \mathrm{MPa}$ with an energy 
cutoff of $500 \mathrm{eV}$ and $3 \times 7 \times 3 \mathrm{k}$-mesh until satisfying $10^{-5} \mathrm{eV} \mathrm{pm}^{-1}$ force tolerance. Supercells $(1 \times 3 \times 1)$ of the optimized structures were used to calculate the phonon modes and thermodynamic functions of $\lambda-\mathrm{Ti}_{3} \mathrm{O}_{5}$ and $\beta-\mathrm{Ti}_{3} \mathrm{O}_{5}$, which were calculated by the direct method implemented in Phonon code with $2 \mathrm{pm}$ displacements using the optimized structures.

Thermodynamic analysis. In the Slichter and Drickamer mean-field model, the Gibbs free energy of the system is described as $G=x(\Delta H)+\gamma x(1-x)+T\{R[x$ $\ln x+(1-x) \ln (1-x)]-x(\Delta S)\}+G_{\beta}$, where $x$ is the ratio of the charge-delocalized unit of $\mathrm{Ti}(1)^{3.3+}-\mathrm{Ti}(2)^{3.3+}-\mathrm{Ti}(3)^{3.3+}$ corresponding to $\lambda-\mathrm{Ti}_{3} \mathrm{O}_{5}, \gamma$ is the interaction parameter between $\lambda-\mathrm{Ti}_{3} \mathrm{O}_{5}$ and $\beta-\mathrm{Ti}_{3} \mathrm{O}_{5}$ phases, $G_{\beta}$ is Gibbs free energy of $\beta-\mathrm{Ti}_{3} \mathrm{O}_{5}$ set as the origin of the energies, and $R$ is the gas constant. The observed phase transition was considered to be a metal-semiconductor phase transition between charge-delocalized $\operatorname{Ti}(1)^{3.3+}-\mathrm{Ti}(2)^{3.3+}-\mathrm{Ti}(3)^{3.3+}$ and charge-localized $\mathrm{Ti}(1)^{3.0}+-\mathrm{Ti}(2)^{3.7+}-\mathrm{Ti}(3)^{3.3+}$ systems, which were regarded as $\lambda-\mathrm{Ti}_{3} \mathrm{O}_{5}$ and $\beta-\mathrm{Ti}_{3} \mathrm{O}_{5}$, respectively. The values of $\Delta H=11.5 \mathrm{~kJ} \mathrm{~mol}{ }^{-1}$ and $\Delta S=25.2 \mathrm{~J} \mathrm{~K}^{-1} \mathrm{~mol}^{-1}$, and a suitable value of $\gamma=\gamma_{\mathrm{a}}+\gamma_{\mathrm{b}} f(T)$, where $\gamma_{\mathrm{a}}=14$ $\mathrm{kJ} \mathrm{mol}^{-1}$ and $\gamma_{\mathrm{b}}=1.08 \times 10^{-2} \mathrm{JK}^{-1} \mathrm{~mol}^{-1}$ to be consistent with the observation results, were used. When the external pressure is applied to the sample, $\Delta H$ is perturbed by the pressure-induced change on the $\Delta U$ and $P \Delta V$ terms. Compared with the pressure-induced change on the $P \Delta V$ term, for example, $0.19 \mathrm{~kJ} \mathrm{~mol}^{-1}$ at $P=60 \mathrm{MPa}$, the change on $\Delta U$ evaluated by the first-principles phonon mode calculations is negligibly small, for example, $1 \times 10^{-3} \mathrm{~kJ} \mathrm{~mol}^{-1}$ at $P=60 \mathrm{MPa}$. Thus, $\Delta H$ is controlled by the $P \Delta V$ term in the present system. The pressureinduced change on $\Delta S$ is also very small, for example, $-0.067 \mathrm{~J} \mathrm{~K}^{-1} \mathrm{~mol}^{-1}$ at $P=60 \mathrm{MPa}$, from the results of first-principles phonon mode calculations.

Current-induced phase transition study. Stainless electrodes are attached to $\beta-\mathrm{Ti}_{3} \mathrm{O}_{5}$ pellet by $\mathrm{Ag}$ paste with an adhesion area of $5 \mathrm{~mm}^{2}$ and electric current of $2 \mathrm{~A}$ was flowed $\left(0.4 \mathrm{~A} \mathrm{~mm}^{-2}\right)$ at $298 \mathrm{~K}$. After that, the XRPD pattern of the surface of the pellet was measured.

\section{References}

1. Nayak, A. P. et al. Pressure-induced semiconducting to metallic transition in multilayered molybdenum disulphide. Nat. Commun. 5, 3731 (2014).

2. Takabayashi, Y. et al. The disorder-free non-BCS superconductor $\mathrm{Cs}_{3} \mathrm{C}_{60}$ emerges from an antiferromagnetic insulator parent state. Science 323, 1585-1590 (2009)

3. Avdeev, M. et al. Pressure-induced ferroelectric to antiferroelectric phase transition in $\mathrm{Pb}_{0.99}\left(\mathrm{Zr}_{0.95} \mathrm{Ti}_{0.05}\right)_{0.98} \mathrm{Nb}_{0.02} \mathrm{O}_{3}$. Phys. Rev. B 73, 064105/1-14 (2006)

4. Kolobov, A. V. et al. Understanding the phase-change mechanism of rewritable optical media. Nat. Mater. 3, 703-708 (2004).

5. Wuttig, M. \& Yamada, N. Phase-change materials for rewriteable data storage. Nat. Mater. 6, 824-832 (2007)

6. Ohkoshi, S. et al. Synthesis of a metal oxide with a room-temperature photoreversible phase transition. Nat. Chem. 2, 539-545 (2010).

7. Miyano, K., Tanaka, T., Tomioka, Y. \& Tokura, Y. Photoinduced insulator-tometal transition in a perovskite manganite. Phys. Rev. Lett. 78, 4257-4260 (1997).

8. Fiebig, M., Miyano, K., Tomioka, Y. \& Tokura, Y. Visualization of the local insulator-metal transition in $\mathrm{Pr}_{0.7} \mathrm{Ca}_{0.3} \mathrm{MnO}_{3}$. Science 280, 1925-1928 (1998).

9. Gütlich, P., Hauser, A. \& Spiering, H. Thermal and optical switching of iron(II) complexes. Angew. Chem. Int. Ed. 33, 2024-2054 (1994).

10. Létard, J. F. et al. Light induced excited pair spin state in an iron(II) binuclear spin-crossover compound. J. Am. Chem. Soc. 121, 10630-10631 (1999).

11. Ohkoshi, S. et al. 90-degree optical switching of output second-harmonic light in chiral photomagnet. Nat. Photonics 8, 65-71 (2014).

12. Ohkoshi, S., Imoto, K., Tsunobuchi, Y., Takano, S. \& Tokoro, H. Light-induced spin-crossover magnet. Nat. Chem. 3, 564-569 (2011).

13. Koshihara, S., Tokura, Y., Mitani, T., Saito, G. \& Koda, T. Photoinduced valence instability in the organic molecular compound tetrathiafulvalence-pchloranil (TTF-CA). Phys. Rev. B 42, 6853-6856 (1990).

14. Collet, E. et al. Laser-induced ferroelectric structural order in an organic charge-transfer crystal. Science 300, 612-615 (2003).

15. Ohkoshi, S. \& Tokoro, H. Photomagnetism in cyano-bridged bimetal assemblies. Acc. Chem. Res. 45, 1749-1758 (2012).

16. Yamanouchi, M., Chiba, D., Matsukura, F. \& Ohno, H. Current-induced domain-wall switching in a ferromagnetic semiconductor structure. Nature 428, 539-542 (2004)

17. Ikeda, S. et al. A perpendicular-anisotropy $\mathrm{CoFeB}-\mathrm{MgO}$ magnetic tunnel junction. Nat. Mater. 9, 721-724 (2010).

18. Asamitsu, A., Tomioka, Y., Kuwahara, H. \& Tokura, Y. Current switching of resistive states in magnetoresistive manganites. Nature 388, 50-52 (1997).

19. Farid, M. M., Khudhair, A. M., Razack, S. A. K. \& Al-Hallaj, S. A review on phase change energy storage: materials and applications. Energ. Convers. Manage. 45, 1597-1615 (2004).
20. Sharma, A., Tyagi, V. V., Chen, C. R. \& Buddhi, D. Review on thermal energy storage with phase change materials and applications. Renew. Sust. Energ. Rev. 13, 318-345 (2009).

21. Cao, Q. \& Liu, P. Hyperbranched polyurethane as novel solid-solid phase change material for thermal energy storage. Eur. Polym. J. 42, 2931-2939 (2006)

22. Benson, D. K., Burrows, R. W. \& Webb, J. D. Solid state phase transitions in pentaerythritol and related polyhydric alcohols. Sol. Energ. Mater. 13, 133-152 (1986).

23. Barrio, M., López, D. O., Tamarit, J. L., Negrier, P. \& Haget, Y. Molecular interactions and packing in molecular alloys between nonisomorphous plastic phases. J. Solid State Chem. 124, 29-38 (1996).

24. Busico, V., Carfagna, C., Salerno, V., Vacatello, M. \& Fittipaldi, F. The layer perovskites as thermal energy storage systems. Sol. Energy 24, 575-579 (1980).

25. Li, W. et al. Study of solid-solid phase change of $\left(n-\mathrm{C}_{n} \mathrm{H}_{2 n+1} \mathrm{NH}_{3}\right)_{2} \mathrm{MCl}_{4}$ for thermal energy storage. Thermochim. Acta 326, 183-186 (1999).

26. Nahas, M. K. \& Constable, F. H. Thermal conductivity of mud brick. Nature 142, 837 (1938).

27. Gur, I., Sawyer, K. \& Prasher, R. Searching for a better thermal battery. Science 335, 1454-1455 (2012).

28. Al-Jabri, K. S., Hago, A. W., Al-Nuaimi, A. S. \& Al-Saidy, A. H. Concrete blocks for thermal insulation in hot climate. Cement Concrete Res. 35, 1472-1479 (2005).

29. Slichter, C. P. \& Drickamer, H. G. Pressure-induced electronic changes in compounds of iron. J. Chem. Phys. 56, 2142-2160 (1972).

30. Sato, H. et al. Baddeleyite-type high-pressure phase of $\mathrm{TiO}_{2}$. Science 251, 786-788 (1991).

31. Huang, L., Durandurdu, M. \& Kieffer, J. Transformation pathways of silica under high pressure. Nat. Mater. 5, 977-981 (2006).

32. Karzel, H. et al. Lattice dynamics and hyperfine interactions in $\mathrm{ZnO}$ and $\mathrm{ZnSe}$ at high external pressures. Phys. Rev. B 53, 11425-11438 (1996).

33. Lipinska-Kalita, K. E., Kalita, P. E., Hemmers, O. A. \& Hartmann, T. Equation of state of gallium oxide to $70 \mathrm{GPa}$ : Comparison of quasihydrostatic and nonhydrostatic compression. Phys. Rev. B 77, 094123 (2008).

34. Azuma, M. et al. Colossal negative thermal expansion in $\mathrm{BiNiO}_{3}$ induced by intermetallic charge transfer. Nat. Commun. 2, 347 (2011).

35. Ahart, M. et al. Origin of morphotropic phase boundaries in ferroelectrics. Nature 451, 545-548 (2008).

36. Medvedev, S. et al. Electronic and magnetic phase diagram of $\beta-\mathrm{Fe}_{1.01} \mathrm{Se}$ with superconductivity at $36.7 \mathrm{~K}$ under pressure. Nat. Mater. 8, 630-633 (2009).

37. Tolbert, S. H. \& Alivisatos, A. P. Size dependence of a first order solid-solid phase transition: the wurtzite to rock salt transformation in CdSe nanocrystals. Science 265, 373-376 (1994).

38. Hanfland, M., Syassen, K., Christensen, N. E. \& Novikov, D. L. New highpressure phases of lithium. Nature 408, 174-178 (2000).

39. McMahon, M. I., Nelmes, R. J., Allan, D. R., Belmonte, S. A. \& Bovornratanaraks, T. Observation of a simple-cubic phase of GaAs with a 16-atom basis (SC16). Phys. Rev. Lett. 80, 5564-5567 (1998).

40. Mujica, A., Rubio, A., Muñoz, A. \& Needs, R. J. High-pressure phases of groupIV, III-V, and II-VI compounds. Rev. Mod. Phys. 75, 863-912 (2003).

41. Nelmes, R. J. \& McMahon, M. I. Identity of InSb-II and InSb-III. Phys. Rev. Lett. 77, 663-666 (1996).

42. Crabtree, G. W. \& Lewis, N. S. Solar energy conversion. Phys. Today 60, 37-42 (2007).

43. Cartlidge, E. Saving for a rainy day. Science 334, 922-924 (2011)

44. Grey, I. E., Li, C. \& Madsen, I. C. Phase equilibria and structural studies on the solid solution. J. Solid State Chem. 113, 62-73 (1994).

45. Onoda, M. Phase transitions of $\mathrm{Ti}_{3} \mathrm{O}_{5}$. J. Solid State Chem. 136, 67-73 (1998).

46. Tomuta, D. G., Ramakrishnan, S., Nieuwenhuys, G. J. \& Mydosh, J. A. The magnetic susceptibility, specific heat and dielectric constant of hexagonal $\mathrm{YMnO}_{3}, \mathrm{LuMnO}_{3}$ and $\mathrm{ScMnO}_{3}$. J. Phys.: Condens. Matter 13, 4543-4552 (2001)

\section{Acknowledgements}

We are grateful to Mr Y. Kakegawa, Mr H. Tsunakawa and Mr S. Ohtsuka (The University of Tokyo) for collecting the TEM and SEM images and we also recognize the Cryogenic Research Center and the Center for Nano Lithography and Analysis, The University of Tokyo, which are supported by MEXT. We are grateful to Mr R. Uemura (Nippon Steel and Sumikin Technology Co., Ltd.) for the thermal conductivity measurement and $\mathrm{Mr} \mathrm{T}$. Tomiura and Mr Y. Okada (NIKKISO Co., Ltd.) for the knowledge of the pressure experiment. The present research was supported in part by the CREST project of JST, the Grant-in-Aid for Young Scientists (A), (B), Research Activity Start-up and the NEXT programme from JSPS, the WPI Research Center Initiative for Atoms, Molecules and Materials, SCF for Promoting Science and Technology and APSA from MEXT, Izumi Foundation and Kurata Foundation. M.Y. and N.O. are grateful to JSPS Research Fellowship for Young Scientists. M.Y. is grateful to ALPS and T.N. is grateful to MERIT from MEXT.

\section{Author contributions}

S.O. designed and coordinated this study, contributed to all the measurements and calculations and wrote the paper. H.T. conducted the temperature-dependent XRPD 
measurements, heat capacity measurements and thermodynamic calculations. M.Y. analysed the first-principles phonon mode calculation results and partially wrote the paper. K.I. carried out the Rietveld analyses of the XRPD patterns and heat capacity data analyses. A.N. conducted the Rietveld analyses and prepared the figures. T.N. carried out the pressure- and temperature-dependent XRPD measurements. K.N. carried out the elemental analyses and background research. N.O. carried out the DSC measurements and MEM analyses. F.H. obtained the TEM and SEM images. K.T. contributed to the synthesis and Rietveld analyses. K.C. carried out the first-principles phonon mode calculations. R.M. and K.P. contributed to the interpretation of the results and to the writing of the paper. All the authors commented on the manuscript.

\section{Additional information}

Supplementary Information accompanies this paper at http://www.nature.com/ naturecommunications
Competing financial interests: The authors declare no competing financial interests.

Reprints and permission information is available online at http://npg.nature.com/ reprintsandpermissions/

How to cite this article: $\mathrm{H}$ Tokoro et al. External stimulation-controllable heat storage ceramics. Nat. Commun. 6:7037 doi: 10.1038/ncomms8037 (2015).

\section{(c) (i)}

This work is licensed under a Creative Commons Attribution 4.0 International License. The images or other third party material in this article are included in the article's Creative Commons license, unless indicated otherwise in the credit line; if the material is not included under the Creative Commons license, users will need to obtain permission from the license holder to reproduce the material. To view a copy of this license, visit http://creativecommons.org/licenses/by/4.0/ 